\title{
A Hierarchical Resilience Enhancement Framework for Interdependent Critical Infrastructures
}

\author{
Xing Liu \& Yi-Ping Fang \\ Chair on Risk and Resilience of Complex System \\ Laboratoire Génie Industriel, CentraleSupélec, Université Paris-Saclay \\ 3 Rue Joliot Curie, 91190 Gif-sur-Yvette, France \\ E-mail: yiping.fang@centralesupelec.fr
}

\section{Enrico Zio}

Centre for Research on Risk and Crises (CRC), Mines ParisTech Sophia Antipolis, PSL Research University, 06904 Sophia Antipolis, France;

Dipartimento di Energia - Politecnico di Milano, Via Ponzio 34/3, 20133 Milano, Italy

E-mail: enrico.zio@polimi.it

\begin{abstract}
Resilience is becoming a key concept for risk assessment and safety management of interdependent critical infrastructures (ICIs). This work proposes a resilience enhancement framework for ICIs. With reference to the accidental event, ex-ante and ex-post solutions for enhancing system resilience are analysed and included into a hierarchical model of resilience enhancement strategies (RES). To provide specific resilience enhancement solutions for ICIs, we integrate the hierarchical model with a model predictive control-based dynamic model of ICI system operation. The relationships between the solutions implemented and their impacts on the system parameters are discussed. A multi-objective optimization (MOO) problem is defined, with the objectives of simultaneously minimizing RES cost and maximizing ICIs resilience. The fast non-dominated sorting genetic algorithm NSGA-II is used to solve the MOO problem. For exemplification, a case study is considered, involving interdependent natural gas network and electric power grid. The results show that the resilience enhancement framework is effective in finding optimal RESs for given ICIs.
\end{abstract}

Keywords: Critical infrastructure, Resilience, Control-based dynamic model, Multiobjective optimization 


\section{Introduction}

In recent years, the concept of resilience has increasingly attracted interest in the domains of safety management, risk analysis and reliability engineering, and from practitioners of different industrial sectors [1-3]. The word resilience is originated from the Latin word "resiliere", which means to "bounce back" [1,4]. In the physical sciences, resilience or elasticity refers to the ability of a substance or material to resume its natural shape after deforming by the application of forces $[5,6]$. In ecological systems, resilience is the ability to retrain system identity, to absorb changes and disturbances, retain the same function and relationships between individuals [7]. For engineering systems, e.g., infrastructures, resilience is referred to the ability of the system to sustain or restore its basic functionality following a risk source or an event (even unknown) [8]. The resilience of an engineering system is the result of a number of appropriate protection activities with respect to the occurrence and development of an accident $[1,9]$.

Critical infrastructures (CIs) are engineering systems that provide devices vital for the national economy and the living quality of people: examples are electrical power grids, transportation systems, telecommunication systems, natural gas pipelines, water supply networks, etc. These systems do not function separately, as their efficient operations rely on the services provided by others $[10,11]$. The CIs are designed to live and evolve for a very long time, exposed to a changing environment and subject to disruptions. The interactions and interdependencies among CIs are of different ways and to different degrees [12] and represent "twosided swords" for their operations. On one side, the exchanges of commodities and services make the operations of CIs more efficient; on the other side, they create potential vulnerabilities for the cascading failures (an initial failure originated in one CI propagates to disrupt other connected CIs,) [13,14]. The safety, reliability and resilience of interdependent CIs (ICIs) are, then, very important characteristics to assess and manage $[15,16]$.

The above justifies the attention devoted to modelling, analysing and enhancing the resilience of ICIs $[1,3,6]$. Most resilience enhancement suggestions are tailored to the different applica- 
tions of interest, and as aimed at increasing the robustness of system elements, optimizing the allocation of redundancies, modifying the system topology, adding self-healing mechanisms, improving maintenance or repair scheduling, facilitating structures retrofitting, improving communication quality, enhancing staff training, etc. [17-22]. Most existing approaches are ad hoc, focusing on developing specific types of enhancement for specific CIs mainly from the perspective of optimization. These studies can be categorized under two main lines: i) pre-disruption investment optimization [23-27], aiming at enhancing CIs resilience via optimum preventive measures, e.g. hardening and upgrading vulnerable components or deploying redundancy before a specific disruptive event strikes the system, and ii) postevent emergency response and recovery planning [28-33], aiming at mitigating system loss through emergency responses right after disruptions and, then, restoring a system to normal operation as quickly and efficiently as possible, e.g., through optimum resource allocation and task scheduling. However, these approaches fall short of accounting for the coordination of resilience measures at different stages, and a framework is missing that provides a comprehensive and quantitative scheme to the decision-makers, when they design, upgrade or rebuild CIs for improving the system resilience.

To fill the gap, in this work, we propose a quantitative framework for resilience enhancement of ICIs. We first discuss the options for resilience enhancement strategies (RES) and organize them into a hierarchical model, which is, then, integrated with a control-based dynamic modelling framework for ICIs [34]. Then, we address the RES optimization problem with respect to the minimization of cost and the maximization of the system resilience.

In the hierarchical model, the resilience enhancement strategies are combinations of physical or organizational activities implemented in the different phases of accident evolution. For example, before the onset of an initiating disruptive event, pre-event preparedness and mitigation activities can be arranged to minimize the impact of the disruption and attenuate its consequences $[19,35]$. After the occurrence of the disruptive event, recovery activities can be activated by post-event measures on the basis of the consequence of the event $[28,36]$. Efforts 
in aspects of communication, organization and management can also improve mitigation and restoration activities [22]. Note that the proposed model does not explicitly consider the probabilities of different failure scenarios; instead, it adopts a "safe-to-fail" paradigm [37] by focusing on the capacity of a system to reorganize and recover from disasters. This is in line with the emphasis given to system resilience when facing so-called high-impact low-frequency events, for which it is really difficult, if not impossible, to have meaningful quantifications of the probabilities of those events.

In a previous work by the authors, a control-based ICIs modelling framework has been proposed to describe and predict the dynamic behaviours of ICIs under disruptions [34]. Within the proposed modelling framework, uncertain system parameters affecting system resilience are defined, including system initial conditions, parameters in time dimension and parameters related to the failure-recovery process of vulnerable elements. In this work, the contributions of the factors in the hierarchical model of RESs on the system performance are discussed. The cost functions of resilience enhancement activities are defined. The optimal RES can be formed by formulating a multi-objective optimization (MOO) problem, with the objectives of the minimization of the cost of RES and the maximization of the total resilience $R_{t}$. Considering the nonlinear and nonconvex nature of the proposed MOO problem, it is solved by the fast non-dominated sorting genetic algorithm NSGA-II [38].

The main contributions of the present study are summarized as follows: 1) a hierarchical model is proposed for identifying in a systematic way the various options of resilience enhancement strategies (RES) for ICIS, 2) which is then integrated with a model predictive control-based dynamic framework to model the transient behaviors of ICIs under adverse perturbations; 3 ) a multi-objective optimization model is proposed to find the optimal RES combinations for the considered cost functions, providing a unified framework for analyzing the tradeoffs of different RES options.

The present work is organized as follows. Section 2 introduces the control-based dynamic modelling framework, including the dynamic equations, the uncertain system parameters and 
the resilience metric. Section 3 presents the hierarchical model of RES, where the factors of RES are included and discussed. The cost functions and the correlations between the activities in RES are defined. Section 4 presents the formulation of the multi-objective optimization problem and introduces the NSGA-II algorithm for the solution. In Section 5, the proposed resilience enhancement framework is applied to a case study concerning a gas network and a power network. Finally, Section V concludes the present work with some future perspectives.

\section{Modelling framework}

\subsection{Control-based dynamic model for ICIs}

CIs are often characterized by hierarchical structures that can be decomposed into parts with certain functionalities [39]. We represent ICIs by a directed graph, where the nodes are the subsystems, i.e., components or functional sets of components, and the edges are the directed links between them. Various resources are produced, consumed, stored and transformed in and between the subsystems, through these physical, cyber or logical links. Note that the directions of the flows matter in the present study, as the critical infrastructure systems we considered, i.e., electrical power distribution networks and gas distribution networks, typically can be seen as directed graphs from the substation/sources to the loads. We consider a discretized state space to represent the behaviour of ICIs under nominal conditions and during disruptive events [34]:

$$
\begin{gathered}
x(t+1)=A x(t)+B u(t)+d(t), \\
y(t)=C x(t)
\end{gathered}
$$

where $x=\left[x_{1} \ldots x_{N_{x}}\right]^{\prime} \in R^{N_{x}}$ is the vector of the system states, which are the flow levels on

subsystems and links. $u=\left[u_{1} \ldots u_{N_{u}}\right]^{\prime} \in R^{N_{u}}$ is the vector of the control variables, which are designated system states for the system controllability, $y=\left[y_{1} \ldots y_{N_{y}}\right]^{\prime} \in R^{N_{y}}$ is the vector of the system outputs, which are the flow levels received by the users, $d=\left[d_{1} \ldots d_{N_{d}}\right]^{\prime} \in R^{N_{d}}$ is the vector of disturbance variables associated with the losses of the system states due to the 
disruptions. The $N_{x} \times N_{x}$ matrix $A$ represents the information of the system's connectivity, while the $N_{u} \times N_{x}\left(N_{u} \leq N_{x}\right)$ matrix $B$ and the $N_{y} \times N_{x}$ matrix $C$ encode the flow transmission coefficients. Matrix $A$ is obtained from the topology of the system, and matrices $B$ and $C$ are derived from the dynamic equations formulated for each component. The readers can refer to [34] for a detailed explanation of the dynamic rules of the flows associated to the five types of subsystems (i.e., suppliers, buffers, transporters, converters, and consumers).

The values of system states and control variables are limited by the capacities of the nodes and the links. The constraints are formulated as follows:

$$
\begin{aligned}
& 0 \leq x(t) \leq C P_{x}, \\
& 0 \leq u(t) \leq C P_{u},
\end{aligned}
$$

where $C P_{x}$ and $C P_{u}$ take values of the capacities of the corresponding elements, i.e., the nodes or the links, which vary with time due to the degradation, the damage caused by disruptions and the recovery activities that follow. The state variable $x(t)$ for link flows are constrained to be nonnegative since we considered directed network model, as explained before. For the control vector $u(t)$, if we take as an example an electrical battery (buffer) which can be charged and discharged to adjust the output flow, $u(t)$ corresponds to controllable output flow of the battery at time $t$, and the output direction is encoded in the coefficient matrix $B$ (with negative elements). In this manner, the control variable $u(t)$ is always nonnegative.

Considering that the units of the resources in ICIs are different, we represent the level of relative insufficient satisfaction $Y_{i_{y}}$ of user $i_{y}$, by a normalized and non-dimensional variable:

$$
Y_{i_{y}}(t)=\frac{D_{i_{y}}(t)-y_{i_{y}}(t)}{D_{i_{y}}(t)} .
$$

where $D_{i_{y}}(t)$ is the demand of user $i_{y}$ at time $t$. Subject to the dynamic equations and constraints, we use Model Predictive Control (MPC) to solve the control inputs at each time step. The MPC approach realizes a finite-horizon optimization by determining sequences of system states and control operations over a prediction horizon $N_{q}$ for the minimization of the 
objective function at each time step within $N_{q}$, and, then, implementing only the first control action [40].

Based on the dynamic model [34], the objective function is formulated to minimize the weighted sum of the insufficient satisfaction function $Y_{i_{y}}(t)$ of users $i_{y}$, within the time hori$\operatorname{zon} N_{q}$ :

$$
\min _{u} \sum_{q=0}^{N_{q}}\left(\sum_{i_{y}=1}^{N_{y}} \omega_{i_{y}} Y_{i_{y}}(t+q \mid t)\right),
$$

where, $\omega_{i_{y}}$ is the weight assigned to the user $i_{y}$, and $\sum_{i_{y}} \omega_{i_{y}}=1$.

By solving the optimization problem with MPC, the control action $u(t \mid t)$ is obtained from the control sequence:

$$
u \triangleq\left\{u(t \mid t), u(t+1 \mid t), \ldots, u\left(t+N_{q}-1 \mid t\right)\right\}
$$

as the result of the optimization problem. Then, only the first control action $u(t \mid t)$ is introduced in the recursion to calculate the system states at $t+1$. More details about the controlbased dynamic model for ICIs can be found in [34].

\subsection{Failure-recovery process and system parameters}

To describe the flow evolution under disruptions, we introduce the system failure-recovery process under a generic external threat and associated system parameters, including parameters in the time dimension, parameters related to the failure-recovery process of the vulnerable elements and system initial conditions. The uncertainty of these system parameters emerges within the modelling framework due to insufficient or imprecise observations and the subjectivity of judgment from experts, etc.

Figure 1 shows the failure-recovery curve for a system element $i$, i.e., a node or a link. In the nominal operation mode, the capacity of the element $i$ is at the maximum of its state, and remains stable at a fixed value; we denote $C P_{i}^{N}$ as the nominal value capacity of element $i$. 


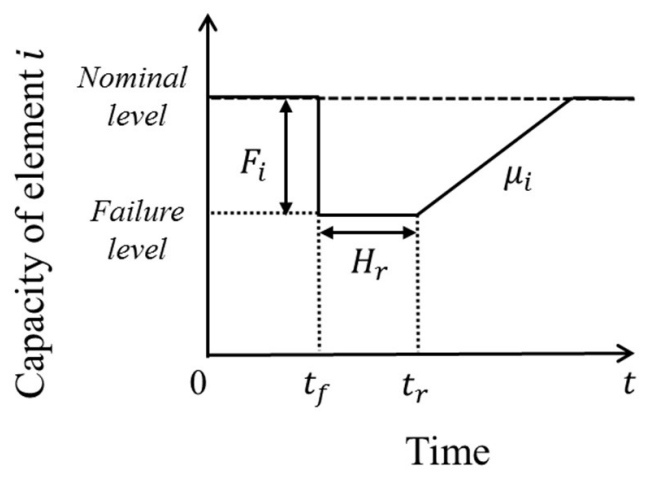

Figure 1. Evolution of the capacity of element $i$

At time $t_{f}$, an initiating disruptive event occurs, which results in the failure of element $i$ of the ICIs. As a consequence of the failure, the nominal capacity element $i$, e.g., $C P_{i}^{N}$, reduces to a degraded state with reduction amount $F_{i}$. Then, the response time $H_{r}=t_{r}-t_{f}$ reflects the rapidity of the failure detection and preparedness of emergency actions of the system [41]. Thanks to the continuous state representation of the elements in our model, we are able to consider a more realistic failure and recovery process and not limit to a binary state process. For example, the recovery process of a failed element $i$ may be described by a linear function with a rate $\mu_{i}$, which depends on the effectiveness of the recovery activities:

$$
C P_{i}(t)=C P_{i}^{N}-F_{i}+\mu_{i}\left(t-t_{r}\right)
$$

where

$$
\begin{gathered}
F_{i}=\left\{\begin{array}{cc}
0 & \text { for } 0 \leq t<t_{f} \\
\text { magnitude of failure } & \text { for } t \geq t_{f}
\end{array},\right. \\
\mu_{i}=\left\{\begin{array}{cc}
0 & \text { for } 0 \leq t<t_{r} \\
\text { recovery rate } & \text { for } t \geq t_{r}
\end{array}\right.
\end{gathered}
$$

The capacities of the system elements are expected to reach their original levels at the end of the restoration activities, i.e., $C P_{i}(t) \leq C P_{i}^{N}$ for all $t \in\left[t_{r}, t_{h}\right]$. Depending on the application, other nonlinear recovery functions also can be considered to describe the recovery process of the elements. For example, the recovery speed of components in freight systems goes slower and slower, as the easy part is recovered at first and the hard part later [42]. For the sake of simplicity of illustration, here, we use linear recovery functions. 
The failure magnitude $F_{i}$ and recovery rate $\mu_{i}$ of vulnerable element $i$ are considered as uncertain variables as well, to reflect the flexibility of the decision on system resilience strategies. The failure magnitude $F_{i}$ is limited to the interval $\left[F_{i_{\text {min }}}, F_{i_{\text {max }}}\right]=\left[0, C P_{i}^{N}\right]$. The recovery rate $\mu_{i}$ varies in $\left[\mu_{i_{\text {min }}}, \mu_{i_{\text {max }}}\right]$, with $\mu_{i_{\text {min }}}=F_{i_{\text {min }}} / H_{h_{\text {max }}}$ and $\mu_{i_{\text {max }}}=F_{i_{\text {max }}} / H_{h_{\text {min }}}$. Moreover, buffering subsystems in the ICIs contribute to system performance by storing resources (for those resources which can be stored), adjusting the supply of resources in nominal operation and compensating the insufficiency of resources in case of shortage during disruptive events. To include the functionality of the buffers, we consider the initial inventory levels of buffers, i.e., $x_{B F_{i}}^{t=0}$, which represents the initial resource level of buffer $B F_{i}$, at $t=0$.

\subsection{System resilience metrics}

The performance of the CIs can be defined from different perspectives (reliability, availability, safety, economics, etc.), e.g., counting the number of operating components [43], the economic loss associated to the components and the casualties of people during the disaster [21]. In all generality, the ICIs aim at providing stable and reliable services to the users, and a measure of their resilience should relate to how they enable and enhance daily life [1]. Therefore, we evaluate the actual performance function of ICIs, $P(t)$, in terms of the weighted sum of the states of users, i.e., the flow levels received by users:

$$
P(t)=\sum_{i_{y}=1}^{N_{y}} \omega_{i_{y}} y_{i_{y}}(t) .
$$

The performance reference function of the ICIs, $P R(t)$, is characterized as the weighted sum of the users' demands:

$$
P R(t)=\sum_{i_{y}=1}^{N_{y}} \omega_{i_{y}} D_{i_{y}}(t) .
$$

Under nominal operating conditions, the supply to each user, i.e., $i_{y}$, with respect to its demand $D_{i_{y}}$ is always achieved, i.e., $y_{i_{y}}(t)=D_{i_{y}}(t)$ and, thus, $P(t)$ maintains values identical to the performance reference function $P R(t)$. 
The shape of the system performance curve describing resilience is often illustrated as triangular or trapezoidal $[21,36,43]$, as shown in Figure 1.

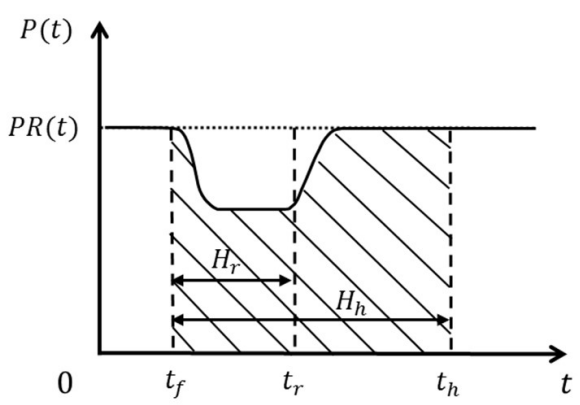

Figure 2. System performance following the occurrence of a disruptive event

In a disruption event, besides the critical response time $H_{r}=t_{r}-t_{f}$, another critical time is $H_{h}=t_{h}-t_{f}$, i.e., the time horizon of system recovery, where $t_{h}$ is the instant when the system performance is expected to return to the nominal level. Note that both $H_{r}$ and $H_{h}$ are uncertain parameters and are functions of the resilience enhancement strategies (RES) implemented, as we discuss in Section 3.

The overall level of system resilience, i.e., the total resilience $R_{t}$, is defined as the proportion of the total area between the actual system performance function $P(t)$ and the time axis (the area shaded with downward diagonal stripes in Figure 2, to the square area between the performance reference function $P R(t)$ and the time axis, for the time period $t_{f} \leq t \leq t_{h}$ with $t_{h} \geq t_{f}$, as shown in Figure 2:

$$
R_{t}=\frac{\int_{t_{f}}^{t_{h}} P(t) d t}{\int_{t_{f}}^{t_{h}} P R(t) d t} .
$$

We point out that many resilience metrics exist in the literature $[1,32,44]$. Yet, the focus of the present study is to provide a comprehensive and quantitative scheme to assist the decision-makers when they design, upgrade or rebuild ICIs for improving system resilience, and different resilience metrics can be incorporated into the proposed framework. 


\section{Hierarchical model of resilience enhancement strategies for ICls}

\subsection{Resilience enhancement strategies}

Different disruptive events may occur in ICIs and some of them are unpredictable and unavoidable, such as malicious attacks and extreme natural disasters. ICIs are engineering systems, thus, their system resilience is not an inherent and spontaneous feature like in ecological systems, but relies on appropriate protection activities (Hosseini et al. 2016; Francis \& Bekera 2014). Various approaches and techniques have been proposed from different perspectives to improve system resilience of ICIs $[1,3,6]$.

To exploit the factors contributing to the resilience enhancement, we propose a hierarchical model of RES as shown in Figure 3. This hierarchical model has multiple layers, where strategic goals, tactical principles, precise activities and interactions among them are displayed.

In the second layer of the hierarchical model, the goal of resilience enhancement is decomposed into mitigation and recovery, which are two main system capacities for enabling resilience. Also, they refer to the two different phases experienced by the system during disruptive events [45]. The mitigation capacity is the ability of the system to resist disorder [46], and is the complement of the system vulnerability to hazards [47]. Before the onset of the initiating disruptive event, or activated at the beginning of the disruption propagation phase, the mitigation activities are arranged to minimize the impacts of the disruption and attenuate its consequences [35]. Recovery activities are, instead, implemented as post-event measures, after the occurrence of the disruptive event, to steer the system performance quickly from adverse consequences and bring it back to the nominal level, at least $[22,48]$.

In the third layer, there are the four resilience enhancement principles for achieving the mitigation of undesirable impacts from the disruptions and the restoration in the aftermath of the disruptions. The two important principles for building the mitigation capacity of a system are increasing the effectiveness of system response to disruptions and reducing the impacts of disruptive events. For example, in electrical power distribution systems, the duration of an 
outage is highly related to the effectiveness of the power grid company's response [49]. The reduction of the consequence of disruptions refers to the reduction in the range of influence and severity of consequences of the disruptions [50,51]. Enhancing system recoverability amounts to improving the capability of the system to restore its performance within a short time and at a low cost [45]. In the aftermath of a disruption, optimal recovery decisions are sought to return the system performance back to its nominal level $[4,25,30]$. Strengthening organization and coordination capacity is another important principle for the improvement of system resilience throughout disruptive events, although its effects are sometimes indirect $[50,52]$.

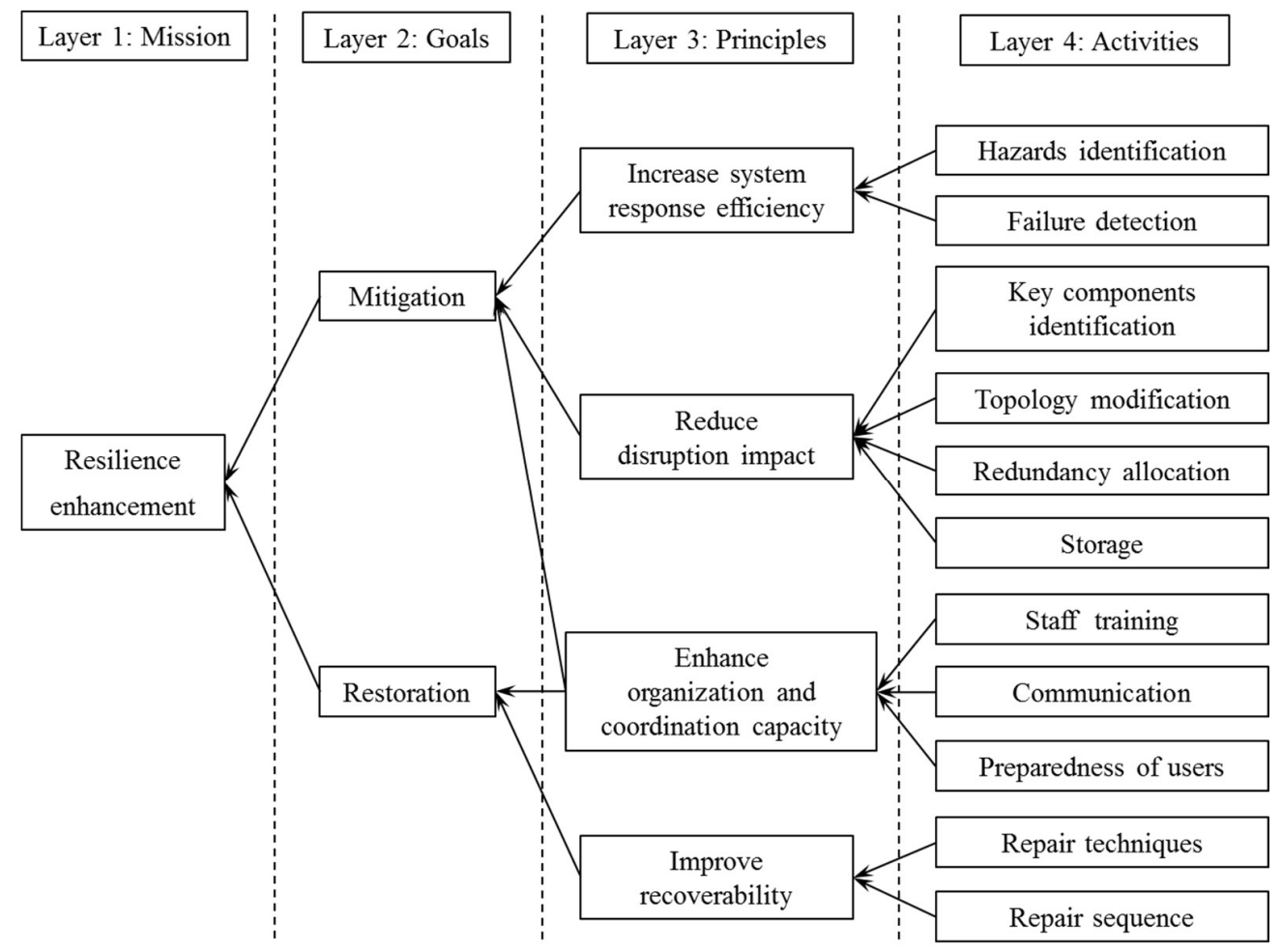

Figure 3. The hierarchical model of resilience enhancement strategies

In the last layer, we include the actions/activities which need to be put in place and executed for the achievement of the third layer principles. The effectiveness of the system's response is supported by hazard identification [53] and early detection of failure [54]. To achieve disruption reduction, adequate prevention measures can be allocated once vulnerable are- 
as/elements have been identified [55]. The modification of topology can be effective to reduce the impact of disruptions [56]. For example, in elected power distribution systems, looped configurations can prevent the propagation of disturbances more than the radial distributions when a component fails [49]. The allocation of redundancies and implementation of appropriate storages (for the CIs which produce and transport storable resources, such as water, gas and electricity in the case of renewable energy systems), are also activities that can serve to reduce the consequences of disruptions $[21,48]$. In the organisational aspect, staff training can enhance the situational awareness and, then, the efficacy of operations [52]; better quality of communication within and between infrastructures have a significant effect on the consequences of disruptive events [48]; for the users, increasing their emergency awareness, e.g., reducing their expectations/needs after the occurrence of adverse events, and enhancing their emergency preparedness, e.g., hospitals hold small-scaled storages of resources that will be used in case of disruptive events, can improve system resilience [49]. To improve system recoverability, we can improve repair techniques to increase the repair rates of included components [28] and optimize the repair sequence $[4,57,58]$.

The proposed hierarchical model includes the main principles and associated representative activities in the RES. Note that the model stands for any arbitrary external disruption, as the ICIs are continually facing new forms of stresses and challenges from changing environments.

\subsection{Effects and costs of resilience enhancement actions on ICIs}

A RES is a combination of activities implemented at the local level. To combine the hierarchical model of RESs with the MPC-based dynamic modelling framework for ICIs, the system parameters need to be associated with the effect of the resilience enhancement activities. Although there exist some literature on the costs of system maintenance, reliability or protection strategies and their effects [59-61], the studies of the correlations between the cost of resilience strategies and their effects are relatively rare, perhaps owing partly to the unpredictable timing of events that challenge resilience, as well as to the difficulties in gaining ac- 
cess to data from organizations concerning their responses to these events. In fact, these relationships or correlations can be analysed and quantified through expert-based approaches which convert and aggregate expert judgements into numerical values $[62,63]$ or statistical analysis based on empirical field data [64]. Although these methods are out of the scope of this paper, for the sake of an example, we list activities and impacts on system parameters in Table I.

Economical costs of these activities are constraints of RES. To find the optimal system resilience enhancement strategies, it is necessary to balance the expenditures of current actions and the potential losses from future disruption occurrences [55]. We define the cost functions

TABLE I. Effects of resilience enhancement activities on ICIs

\begin{tabular}{|c|c|c|}
\hline Activities & Impacts of activities & Affected parameters \\
\hline Hazards identification & $\begin{array}{l}\text { Aid to the prediction and early awareness } \\
\text { of potential accidents. }\end{array}$ & $H_{r}, F_{i}$ \\
\hline Failure detection & $\begin{array}{l}\text { Detect the position and magnitude of } \\
\text { failure efficiently and accurately. }\end{array}$ & $H_{r}$ \\
\hline $\begin{array}{l}\text { Key components identi- } \\
\text { fication }\end{array}$ & $\begin{array}{l}\text { Identify the key components, for protect- } \\
\text { ing them. }\end{array}$ & $F_{i}$ \\
\hline Topology modification & Increase the robustness of elements. & $F_{i}$ \\
\hline Redundancy allocation & Increase the robustness of elements. & $F_{i}$ \\
\hline Storage & Increase the storage of buffer nodes. & $x_{B F_{i}}^{t=0}$ \\
\hline Staff training & Increase the efficiency of operators. & $F_{i}, H_{r}, \mu_{i}$ \\
\hline Communication & $\begin{array}{l}\text { Establish efficient communication channels } \\
\text { for operators. }\end{array}$ & $F_{i}, H_{r}, \mu_{i}$ \\
\hline Preparedness of users & Increase the tolerance of users. & $H_{h}$ \\
\hline Repair techniques & $\begin{array}{l}\text { Improve repair efficiency of failed ele- } \\
\text { ments. }\end{array}$ & $\mu_{i}$ \\
\hline Repair sequence & $\begin{array}{l}\text { Improve repair efficiency of failed ele- } \\
\text { ments. }\end{array}$ & $\mu_{i}$ \\
\hline
\end{tabular}


for each activity $v$. The cost function of an activity has two parts, standard and supplementary costs:

$$
c_{i_{v}}=c_{i_{v}}^{f}+c_{i_{v}}^{s}
$$

The standard cost of a certain activity, i.e., $c_{i_{v}}^{f}$, is a fixed value referring to the base investment cost and operation and maintenance costs [65]. The standard cost allows maintaining the standard effect of the activity, e.g., the cost of the failure detection system ensures that the response time reaches its average level $\left(H_{r}^{a}=\frac{H_{r}^{\max }+H_{r}^{\min }}{2}\right)$.

The supplementary costs of the RES activities, i.e., $c_{i_{v}}^{s}$, determine the effects of the extra effort of an activity. It is really challenging, if not possible, to derive a generic costeffectiveness relation for the various RES activities of interest. For the sake of simplicity, in the present study we consider that the effects of activities vary approximately linearly with the supplementary costs in a certain range, i.e., truncated affine functions. This can be regarded as a linearized version of the widely used S-shaped utility function [66]. Assuming that there are $N_{p}$ system parameters, there are two possible cases for a system parameter $p_{i_{p}}$, $i_{p} \in\left\{1, \ldots, N_{p}\right\}:$

1) for the system parameters which monotonically increase with costs:

$$
p_{i_{p}}=p_{i_{p}}^{a}+\left(p_{i_{p}}^{\max }-p_{i_{p}}^{a}\right) \cdot \sum_{i_{v}} \tau_{p_{i_{p}}}^{i_{v}} \cdot \frac{c_{i_{v}}^{s}}{{C_{i_{v}}^{s}}^{\text {max }}-C_{i_{v}}^{\text {min }}} .
$$

2) for the system parameters which monotonically decrease with costs

$$
p_{i_{p}}=p_{i_{p}}^{a}-\left(p_{i_{p}}^{\max }-p_{i_{p}}^{a}\right) \cdot \sum_{v_{i}} \tau_{p_{i_{p}}}^{v_{i}} \cdot \frac{c_{i_{v}}^{s}}{{C_{i_{v}}^{s}}^{\text {max }}-C_{i_{v}}^{s i n}} .
$$

where $p_{i_{p}}^{a}$ is the nominal/base level of the system parameter and $\tau_{i_{p}}^{i_{v}}$ is the weight of the contributions of activity $v$ to the system parameter $p_{i_{p}}$, with $\sum_{i_{v}} \tau_{i_{p}}^{i_{v}}=1$, which depends on the level of the contribution of the activities on system parameters. Note that, in reality, the cost functions depend on the precise means accountable for the activities and the quantity of the consumed materials, e.g., the cost of a section of gas pipeline increases with the length and 
diameter. The effects of the activities on the system parameters can be estimated by empirical methods based on field data and/or expert judgement [64]. The proposed cost function aims at providing a simple general formulation so that different types of activities can be considered.

\section{RES multi-objective optimization and NSGA-II solution}

\subsection{RES multi-objective optimization}

Within the system resilience enhancement framework, we incorporate the dynamic model and RES hierarchical model within an optimization problem to identify optimal RES for given ICIs.

We formulate a MOO problem to achieve the balance between a RES, which is a combination of system resilience activities, and its cost of implementation. The decision variables are the supplementary investments/costs of the resilience improvement actions $c_{i_{v}}^{s}$, which are nonnegative continuous variables. Note that we keep the standard investment/cost $c_{i_{v}}^{f}$ fixed for every activity $v, c_{i_{v}}^{s}=0$ represents that no extra effort is devoted to activity $v$. The MOO problem seeks to simultaneously maximize system resilience and minimize the cost of RES implementation. The objective functions to be minimized are

$$
f_{1}=1-R_{t}=1-\frac{\int_{t_{f}}^{t_{h}} \sum_{i_{y}}^{i_{y}=N_{y}} \omega_{i} y_{i_{y}}(t) d t}{\int_{t_{f}}^{t_{h}} \sum_{i_{y}}^{i_{y}=N_{y}} \omega_{i_{y}} D_{i_{y}}(t) d t},
$$

and

$$
f_{2}=\text { Cost }=\sum_{v_{i}} c_{v_{i}}^{s}
$$

To evaluate the resilience of the system, uncertain system parameters, i.e., the response time $H_{r}$, the recovery time $H_{h}$, the initial resource level of buffers $x_{B F_{i}}^{t=0}$, the failure magnitude $F_{i}$ 
and recovery rate $\mu_{i}$ of vulnerable element $i$, are first calculated with equations (14) and (15). The ICI dynamic simulation is performed, based on MPC, following the approach in Section 2 , to obtain the system performance functions. Eventually, the resilience level of system $R_{t}$ is calculated according to equation (12). The cost is obtained by summing the supplementary costs of all activities. The proposed optimization is a non-linear multi-objective problem due to the MPC-based dynamic simulation for computing the system performance in $f_{1}$. To obtain the Pareto front, we adopt a population-based global optimization method, i.e., NSGAII, to solve the proposed MOO problem.

\subsection{Solving procedure of the RES MOO for ICIs}

NSGA-II is a heuristic optimization technique belonging to the class of Evolutionary Algorithms (EAs) and a most effective way of solution for MOO problems [65], also applicable to non-convex and non-smooth problems [38]. In this work, the evaluation of system resilience of ICIs is integrated into the MOO problem. The steps to adopt NSGA-II for solving the proposed MOO can be summarized as follows:

\section{Step 1. Initialization of MOO parameters}

Define the values of: the population size $N P$, the crossover probability $\mathrm{Cr} P$, the mutation probability $M P$, and the maximum number of generations $N_{\text {max }}$;

\section{Step 2. Generation of initial parent population}

Generate randomly the initial parent population $p^{g=0}$, which is a set containing $N P$ vectors. Each vector is also called a chromosome and forms a candidate solution to the optimization problem. Each element of the vector is a randomly created string of binary values, each one associated with a decision variable, i.e., the activity $v$.

Step 3. Generation of intermediate population

Apply the binary tournament selection operator based on the crowed-comparison-operator [38] to the parent population $p^{g}$ and obtain an intermediate population $p^{g \prime}$.

\section{Step 4. Generation of offspring population}


Perform the evolution operations of mutation and crossover on the intermediate population with the respective probabilities and obtain the offspring generation $o^{g}$.

Step 5. Combination of the parent and offspring populations

Combine the parent and offspring populations to obtain a union population $u^{g}=p^{g} \cup o^{g}$.

Step 6. Evaluation

Step 6.1 Evaluation of system behaviour

For each of the chromosomes in the union population $u^{g}$, perform the system dynamic simulation by the MPC model presented in Section 2, and return the dynamic evolution of system states.

Step 6.2 Evaluation of objective functions

Calculate the value of objective functions $f_{1}$ and $f_{2}$ with equations (16) and (17) for each chromosome in $u^{g}$.

Step 7. Selection of parent population of the next generation

Select the first $N P$ chromosomes from the union population $u^{g}$ based on non-domination and crowding distance with respect to their values of objective functions, to be the parent population of the next generation.

Step 8. Stop the algorithm if the current generation number reaches $N_{\text {max }}$, otherwise go to Step 3.

The process of searching the non-dominated solutions set is shown as in the flowchart of Figure 4 . 


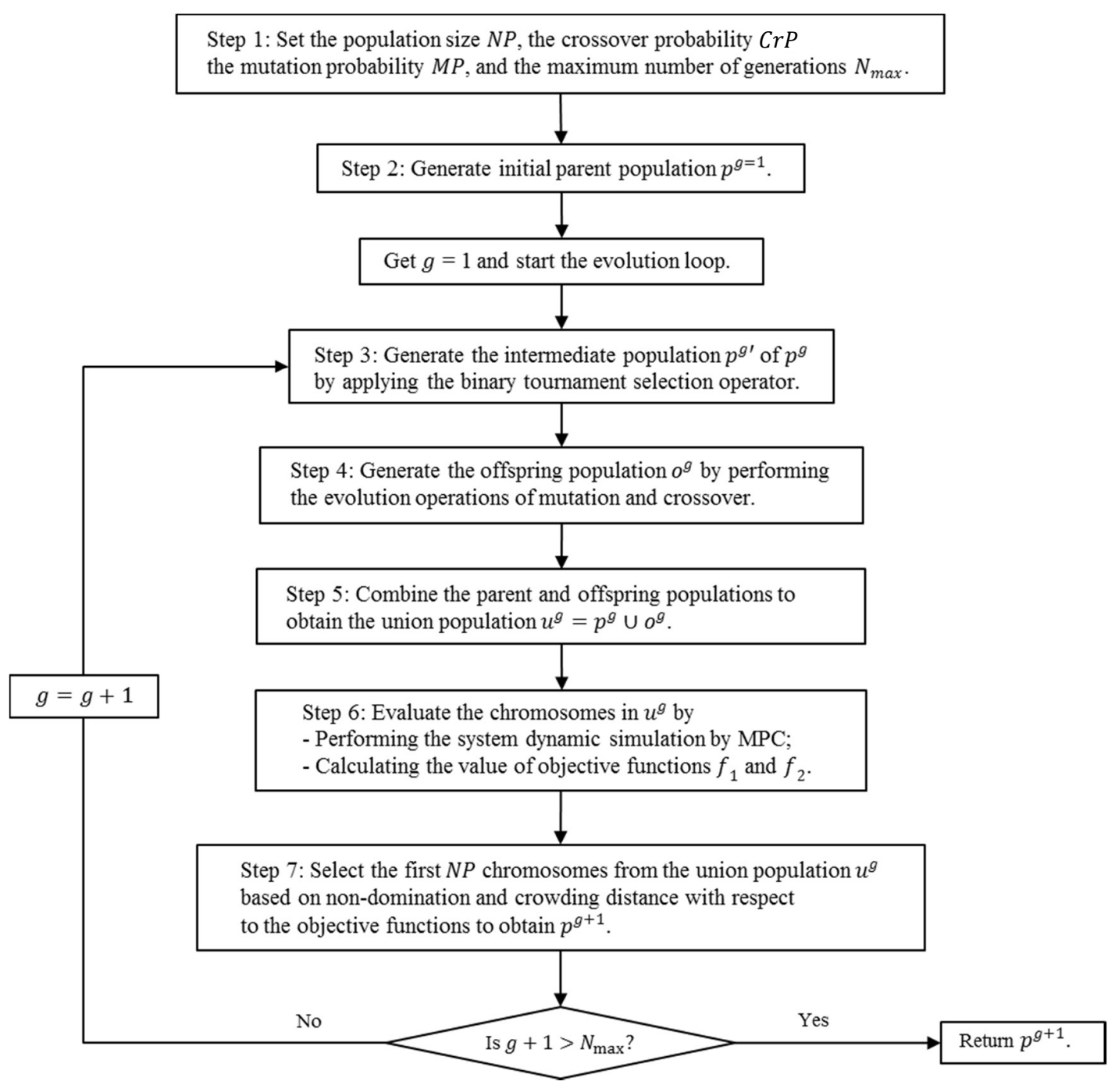

Figure 4. Flowchart of the NSGA-II algorithm for solving the RES MOO problem

\section{Case study and results}

\subsection{Interconnected natural gas distribution network and power grid}

We illustrate a case study of ICIs taken from [67], concerning two interconnected CIs: a natural gas distribution network and a power grid (Figure 5, solid and dash-dotted lines, respectively). The objective of this system is to provide the necessary amount of gas and electricity to the demand nodes. In particular, the gas distribution network supplies gas to two users, $D_{1}$ and $D_{2}$, and to two electric power generators, $E_{1}$ and $E_{2}$, that provide electricity to two 
users of electricity, $L_{1}$, and $L_{2}$.

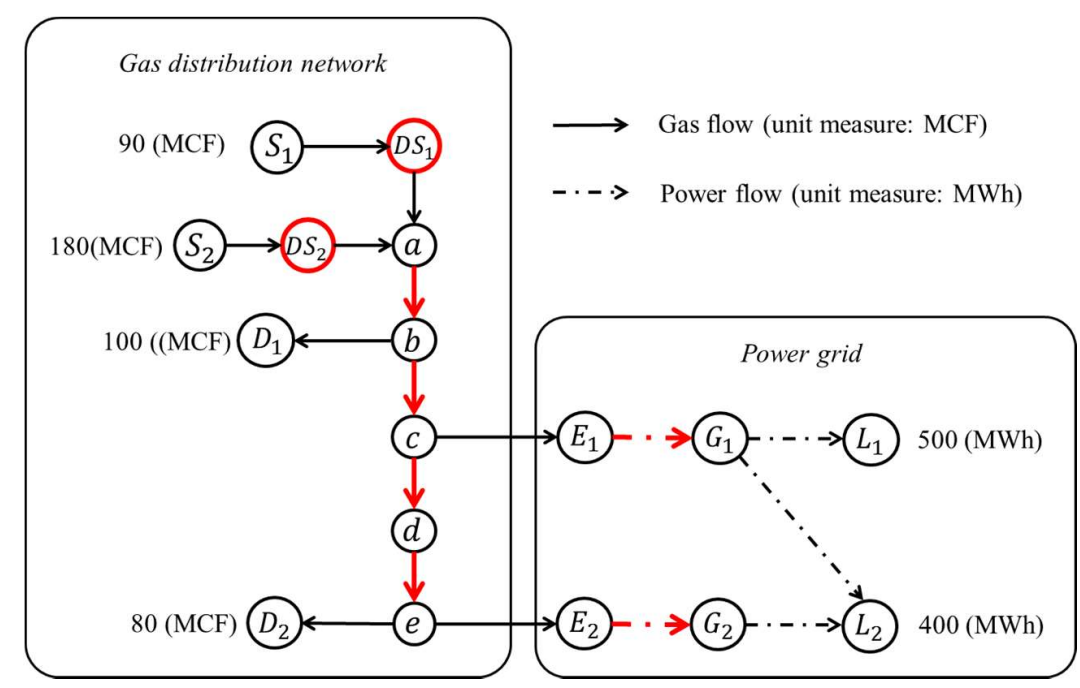

Figure 5 Interconnected natural gas-power systems.

The natural gas distribution network has two suppliers, $S_{1}$ and $S_{2}$, whose outputs are assumed to be equal to $90 \mathrm{MCF}$, i.e., 1000 cubic feet, and $180 \mathrm{MCF}$, respectively; two buffers (gas reservoirs), $\mathrm{DS}_{1}$ and $\mathrm{DS}_{2}$; five transporters $a, b, c, d$ and $e$; and two users $D_{1}$ and $D_{2}$, whose demands, $D_{D 1}$ and $D_{D 2}$, are equal to $100 \mathrm{MCF}$ and $80 \mathrm{MCF}$, respectively. The electric power network has two converters (electric power generators), $E_{1}$ and $E_{2}$, that transform gas into electricity with a constant coefficient $\beta$, where $\beta=10 \mathrm{MWh} / \mathrm{MCF}$, i.e., $1 \mathrm{MCF}$ of natural gas produces $10 \mathrm{MWh}$ of electricity; two transporters, $G_{1}$ and $G_{2}$; and two users $L_{1}$ and $L_{2}$, whose demands, $D_{L 1}$ and $D_{L 2}$, are equal to $500 \mathrm{MWh}$ and $400 \mathrm{MWh}$, respectively.

In this case study, $20 \mathrm{IVs}$ (i.e., system design parameters, failure magnitudes and recovery rates of these vulnerable elements) affecting the system resilience are defined, and their minimum (min), maximum (max), and nominal (nom) values $\left(p_{i_{p}}^{a}\right)$ are given in Table II.

TABLE II. Ranges of uncertain system parameters

\begin{tabular}{llll}
\hline \hline Description of system parameters & Symbol & {$[$ min, nom, max $]$} & Unit measure \\
\hline Response time & $H_{r}$ & {$[0,15,30]$} & hrs \\
\hline \hline
\end{tabular}




\begin{tabular}{|c|c|c|c|}
\hline Time horizon & $H_{h}$ & {$[50,85,120]$} & hrs \\
\hline Initial storage of buffer $D S_{1}$ & $x_{D s_{1}}^{t=0}$ & {$[1000,2500,4000]$} & $\mathrm{MCF}$ \\
\hline Initial storage of buffer $D S_{2}$ & $x_{D s_{2}}^{t=0}$ & {$[2000,5000,8000]$} & $\mathrm{MCF}$ \\
\hline Failure magnitude of supplier $S_{1}$ & $F_{1}$ & {$[0,45,90]$} & $\mathrm{MCF}$ \\
\hline Recovery rate of supplier $S_{1}$ & $\mu_{1}$ & {$[0,0.9,1.8]$} & $\mathrm{MCF} / \mathrm{hrs}$ \\
\hline Failure magnitude of supplier $S_{2}$ & $F_{2}$ & {$[0,90,180]$} & $\mathrm{MCF}$ \\
\hline Recovery rate of supplier $S_{2}$ & $\mu_{2}$ & {$[0,1.8,3.6]$} & $\mathrm{MCF} / \mathrm{hrs}$ \\
\hline Failure magnitude of link $L_{a-b}$ & $F_{3}$ & {$[0,150,300]$} & $\mathrm{MCF}$ \\
\hline Recovery rate of link $L_{a-b}$ & $\mu_{3}$ & {$[0,3,6]$} & $\mathrm{MCF} / \mathrm{hrs}$ \\
\hline Failure magnitude of link $L_{b-c}$ & $F_{4}$ & {$[0,85,170]$} & $\mathrm{MCF}$ \\
\hline Recovery rate of link $L_{b-c}$ & $\mu_{4}$ & {$[0,1.7,3.4]$} & $\mathrm{MCF} / \mathrm{hrs}$ \\
\hline Failure magnitude of link $L_{c-d}$ & $F_{5}$ & {$[0,50,100]$} & $\mathrm{MCF}$ \\
\hline Recovery rate of link $L_{c-d}$ & $\mu_{5}$ & {$[0,1,2]$} & $\mathrm{MCF} / \mathrm{hrs}$ \\
\hline Failure magnitude of link $L_{d-e}$ & $F_{6}$ & {$[0,50,100]$} & $\mathrm{MCF}$ \\
\hline Recovery rate of link $L_{d-e}$ & $\mu_{6}$ & {$[0,1,2]$} & $\mathrm{MCF} / \mathrm{hrs}$ \\
\hline Failure magnitude of link $L_{E_{1}-G_{1}}$ & $F_{7}$ & {$[0,400,800]$} & MWh \\
\hline Recovery rate of link $L_{E_{1}-G_{1}}$ & $\mu_{7}$ & {$[0,8,16]$} & MWh/hrs \\
\hline Failure magnitude of link $L_{E_{2}-G_{2}}$ & $F_{8}$ & {$[0,200,400]$} & MWh \\
\hline Recovery rate of link $L_{E_{2}-G_{2}}$ & $\mu_{8}$ & {$[0,4,8]$} & MWh/hrs \\
\hline
\end{tabular}

\subsection{Results}

\subsubsection{Resilience enhancement activities}

In this case, only the most relevant system parameters, i.e., $F_{3}, F_{7}, F_{2}, F_{4}, H_{r}, \mu_{3}$ and $H_{h}$, identified by a previous sensitivity analysis [34], are considered variant to the RES activities; The other parameters are set as their nominal values in Table II. Note that this setting largely mitigates the complexity of the optimization problem, allowing the analysis to focus on the most important parameters. According to the hierarchical model, we identify the relation- 
ships between the RES activities and the system parameters, as shown in Table III.

TABLE III. RES activities affecting system parameters

\begin{tabular}{|c|c|c|c|}
\hline$i_{v}$ & Activities $v$ & Affected parameters $p_{i_{p}}$ & Contribution \\
\hline \multirow{2}{*}{1} & \multirow{2}{*}{ Identify and predict potential hazards } & $F_{3}, F_{7}, F_{2}, F_{4}$ & Secondary \\
\hline & & $H_{r}$ & Secondary \\
\hline 2 & Improve the efficiency of failure detection & $H_{r}$ & Primary \\
\hline 3 & $\begin{array}{l}\text { Identify and improve the maintenance of } \\
\text { key elements }\end{array}$ & $F_{3}, F_{7}, F_{2}, F_{4}$ & Secondary \\
\hline 4 & Design redundancy for link $L_{a-b}$ & $F_{3}$ & Primary \\
\hline 5 & Design redundancy for link $L_{E_{1}-G_{1}}$ & $F_{7}$ & Primary \\
\hline 6 & Design redundancy for buffer $D S_{2}$ & $F_{2}$ & Primary \\
\hline 7 & Design redundancy for link $L_{b-c}$ & $F_{4}$ & Primary \\
\hline \multirow[b]{3}{*}{8} & \multirow{4}{*}{ Staff training } & $F_{3}, F_{7}, F_{2}, F_{4}$ & Secondary \\
\hline & & $H_{r}$ & Secondary \\
\hline & & & Secondary \\
\hline \multirow[b]{2}{*}{9} & & $H_{r}$ & Secondary \\
\hline & $\begin{array}{l}\text { Establish efficient communication chan- } \\
\text { nels for operators }\end{array}$ & $\mu_{3}$ & Secondary \\
\hline 10 & Emergency education for users & $H_{h}$ & Primary \\
\hline 11 & Improve repair efficiency for link $L_{a-b}$ & $\mu_{3}$ & Primary \\
\hline
\end{tabular}

In the last column of Table III, we comment on the level of contribution that an activity has for improving the corresponding parameters in the considered ICIs. An activity is labeled as 
"primary" if its implementation can improve solely the affected parameters with a reasonably high probability; otherwise, it is labeled as "secondary". For example, the activity "improving the efficiency of failure detection" will likely reduce the response time $H_{r}$, and can thus be considered as a "primary" contributor; other activities such as "identify and predict potential hazards", "staff training" and "establish efficient communication channels for operators" can indeed, partially contribute to the reduction of the response time, but their impacts are either indirect or insufficient. In reverse, the RES activities related to each system parameter of ICIs can be obtained as shown in Table IV. In this example, we arbitrarily assign a contribution weight $\tau_{i_{p}}^{i_{v}}=0.7$ for the activities that have primary contributions to the system parameters and the rest of weights values (since $\sum_{i_{v}} \tau_{i_{p}}^{i_{v}}=1$ ) is equally distributed to the activities that have secondary contributions.

TABLE IV. System parameters affected by RES activities

\begin{tabular}{|c|c|c|c|c|}
\hline$i_{p}$ & $p_{i_{p}}$ & $i_{v}$ & Activities $v$ & $\tau_{i_{p}}^{i_{v}}$ \\
\hline \multirow{4}{*}{1} & \multirow{4}{*}{$F_{3}$} & 1 & Identify and predict potential hazards & 0.1 \\
\hline & & 3 & Identify and improve maintenance of key elements & 0.1 \\
\hline & & 4 & Design redundancy for link $L_{a-b}$ & 0.7 \\
\hline & & 8 & Staff training & 0.1 \\
\hline \multirow{4}{*}{2} & \multirow{4}{*}{$F_{7}$} & 1 & Identify and predict potential hazards & 0.1 \\
\hline & & 3 & Identify and improve maintenance of key elements & 0.1 \\
\hline & & 5 & Design redundancy for link $L_{E_{1}-G_{1}}$ & 0.7 \\
\hline & & 8 & Staff training & 0.1 \\
\hline \multirow{4}{*}{3} & \multirow{4}{*}{$F_{2}$} & 1 & Identify and predict potential hazards & 0.1 \\
\hline & & 3 & Identify and improve maintenance of key elements & 0.1 \\
\hline & & 6 & Design redundancy for buffer $D S_{2}$ & 0.7 \\
\hline & & 8 & Staff training & 0.1 \\
\hline 4 & $F_{4}$ & 1 & Identify and predict potential hazards & 0.1 \\
\hline
\end{tabular}




\begin{tabular}{|c|c|c|c|c|}
\hline & & 3 & Identify and improve maintenance of key elements & 0.1 \\
\hline & & 7 & Design redundancy for link $L_{b-c}$ & 0.7 \\
\hline & & 8 & Staff training & 0.1 \\
\hline \multirow{4}{*}{5} & \multirow{4}{*}{$H_{r}$} & 1 & Identify and predict potential hazards & 0.1 \\
\hline & & 2 & Improve the efficiency of failure detection & 0.7 \\
\hline & & 8 & Staff training & 0.1 \\
\hline & & 9 & Establish efficient communication channels for operators & 0.1 \\
\hline \multirow{3}{*}{6} & \multirow{3}{*}{$\mu_{3}$} & 8 & Staff training & 0.15 \\
\hline & & 9 & Establish efficient communication channels for operators & 0.15 \\
\hline & & 11 & Improve repair efficiency for link $L_{a-b}$ & 0.7 \\
\hline 7 & $H_{h}$ & 10 & Emergency education of users & 1 \\
\hline
\end{tabular}

In the MOO problem, the decision variables are the supplementary costs of the activities in RES, $c_{i_{v}}^{s}$, as shown in Table III. To simplify the calculation, we consider the normalized value for the supplementary cost of resilience improvement activities, with $c_{i_{v}}^{s}$ max $=1$ and $c_{i_{v}}^{s i n}=$ 0, i.e., the limits of decision variables in the MOO problem.

\subsubsection{Multi-objective optimization}

The parameters of the NSGA-II for the MOO solution are: the population size $N P=50$, crossover probability $C P=0.5$, mutation probability $M P=0.01$, and maximum number of generations $N_{\max }=200$. Following the steps in Section 4.2, the optimal Pareto front is produced, as shown in Figure 6. The simulations are carried out by using Yalmip Toolbox [68] and CPLEX optimizer [69] on MATLAB 2015a, on an Intel® Core $^{\mathrm{TM}} 2$ Duo CPU E7600 @ $3.07 \mathrm{GHz}$. 


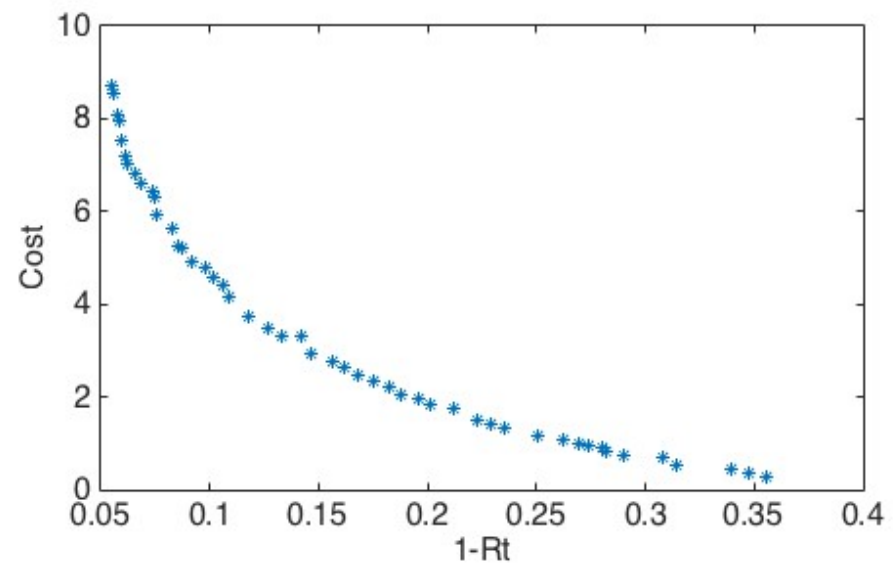

Figure 6. The optimal Pareto front

Figure 7 displays three representative optimal solutions of RES chosen from the Pareto front, with three levels of resilience and cost values:

- $\quad \mathrm{PF} 1$ is with the minimum value of $f_{1}$ and the maximum value of $f_{2}$, i.e., the solution of highest resilience but also highest cost, $R_{t}=0.9454$ and Cost $=8.7107$;

- PF 2 is the best compromise solution obtained using the min-max approach [70] to compromise between resilience and cost, $R_{t}=0.8667$ and Cost $=3.3275$;

- $\mathrm{PF} 3$ is with the maximum value of $f_{1}$ and the minimum value of $f_{2}$, i.e., the solution of lowest resilience and lowest cost, $R_{t}=0.6447$ and Cost $=0.7243$.

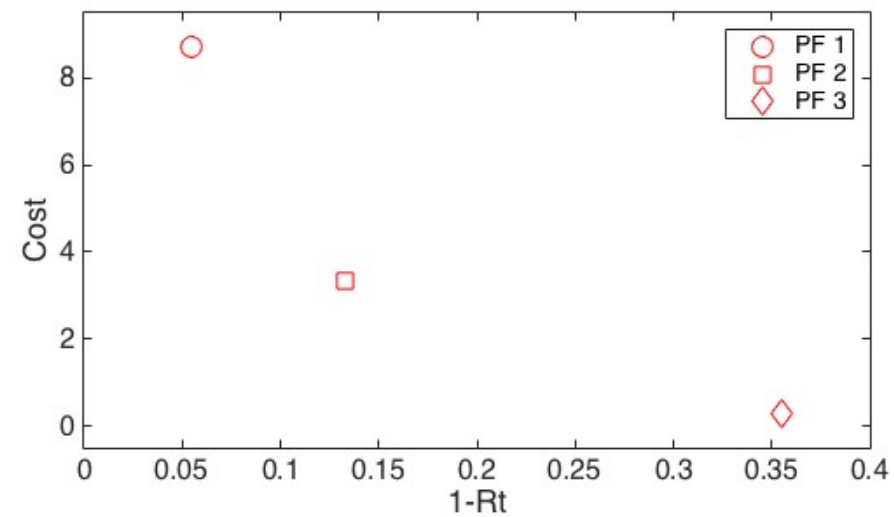

Figure 7. Three representative optimal solutions chosen from the Pareto front

TABLE V. Cost of RES activities associated with the three optimal solutions of Figure 7 


\begin{tabular}{llll}
\hline & & \multicolumn{1}{c}{$c_{i_{v}}^{s}$} & \\
\cline { 2 - 4 } & PF 1 & PF 2 & PF 3 \\
\hline 1 & 0.9912 & 0.0283 & 0.0196 \\
\hline 2 & 0.9863 & 0.9873 & 0.0059 \\
\hline 3 & 0.9599 & 0.0293 & 0 \\
\hline 4 & 0.9453 & 0.0802 & 0.0029 \\
\hline 5 & 0.8690 & 0.6158 & 0.0704 \\
\hline 6 & 0.8866 & 0.1134 & 0.0303 \\
\hline 7 & 0.9316 & 0.6716 & 0.0459 \\
\hline 8 & 0.8935 & 0.0635 & 0.0323 \\
\hline 9 & 0.5259 & 0.0098 & 0.0049 \\
\hline 10 & 0.7146 & 0.7146 & 0.0577 \\
\hline 11 & 0.0068 & 0.0137 & 0.0059 \\
\hline \hline
\end{tabular}

Each optimal solution in the Pareto front represents an optimal RES, i.e., a combination of resilience enhancement activities. Focusing on these the optimal solutions of Figure 7 , the decision variables, i.e., the supplementary costs of the resilience improvement activities, are in Table V. The normalized supplementary investment $c_{i_{v}}^{s}$ might not provide direct guidance for practical implementation. However, this setting is general enough to be applicable in various CI systems and can be used to determine the relative investment/cost for different resilience enhancement actions, e.g., $29.7 \%$ of the total investment should be allocated to $i_{v}=2$ in PF 2 according to Table V. Moreover, the corresponding optimal values of the system variables, i.e., $F_{3}, F_{7}, F_{2}, F_{4}, H_{r}, \mu_{3}$ and $H_{h}$, can be derived, as shown in Table VI. Recall that the physical meanings of the system variables are reported in Table II, e.g., $F_{3}=8.08$ means that link $L_{a-b}$ loses 8.08 MCF of natural gas transmission capacity. By comparing the optimal parameter values to their nominal values in Table VI, one can find that $F_{7}$ is the most reduced one in PF3, indicating that the mitigation RESs aiming at reducing $F_{7}$, i.e., $i_{v}=$ 
$1,3,5,8$, should be given top priority when the available resource is very limited (the lowest cost in PF 3). In the best compromise solution PF 2, the mitigation RESs aiming at reducing $F_{4}$, i.e., $i_{v}=1,3,7,8$, and the response/restoration RESs aiming at reducing $H_{r}$, i.e., $i_{v}=$ $1,2,8,9$, should, then, be emphasized besides $F_{7}$.

TABLE VI. The optimal system parameters associated with the three optimal solutions of Figure 7

\begin{tabular}{ccccc}
\hline \hline & \multicolumn{3}{c}{ Optimal values } & \\
\cline { 2 - 4 }$p_{k}$ & PF 1 & PF 2 & PF 3 & \\
\hline$F_{3}$ & 8.08 & 139.77 & 148.92 & 150 \\
\hline$F_{7}$ & 42.89 & 222.72 & 346.74 & 400 \\
\hline$F_{2}$ & 8.54 & 81.77 & 87.63 & 90 \\
\hline$F_{4}$ & 5.39 & 44.01 & 81.82 & 85 \\
\hline$H_{r}$ & 1 & 5 & 15 & 15 \\
\hline$\mu_{3}$ & 3.65 & 3.07 & 3.03 & 3 \\
\hline$H_{h}$ & 110 & 110 & 87 & 85 \\
\hline \hline
\end{tabular}

We implement the three RESs obtained from the optimal Pareto Front solutions and observe the system performance in three corresponding enhanced scenarios. The evolutions of the performance function of the ICIs in the case of these three optimal RES solutions are displayed in Figure 8. As shown in this Figure, the curves of system performance corresponding to the three solutions are consistent with the results of the MOO. The scenario that implements the RES of PF 1 has the best resilience performance. In this scenario, the lowest level of satisfaction function is the highest among the three scenarios and it is the first to recover to the nominal state. The optimal set of RES in PF 2 achieves a trade-off between the cost and the effects of the improvement of system resilience. The scenario corresponding to the RES of PF 3 is the least resilient (but also the cheapest).

Also, it is shown in Figure 8 that recovery curves have turning points due to different recov- 
ery start and end instants of the users' states and chattering behaviours caused by the randomness of MPC problem solution. One turning point typically represents the end of the recovery of a user, i.e., the demand of the user is satisfied. The recovery start and end instants of the users are related to the importance weights as well as the distances between the users and the failed elements, which are determined by the system topology.

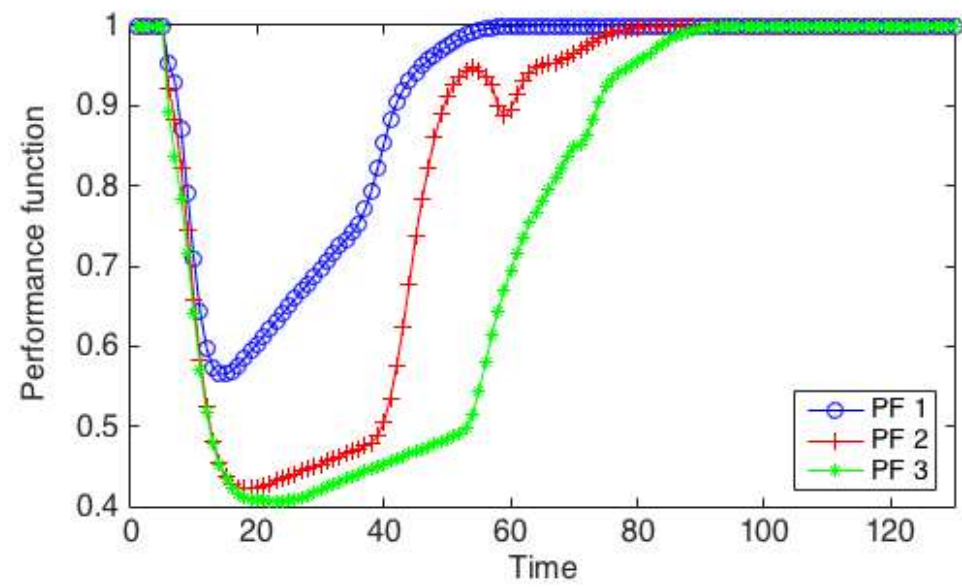

Figure 8. Evolution of the performance functions for three optimal RESs of Figure 7

Note that MOO finds the Pareto-optimal set of solutions, which provide the decision-maker with a comprehensive view of the possible tradeoffs among different objectives. In practice, the decision-maker will select one solution from the Pareto front based on his/her preferences over the different objectives. For instance, one can choose the least costly solution, which gives a certain level of system resilience, e.g., $R_{t} \geq 0.9$.

\section{Conclusion}

In this work, we have proposed a resilience enhancement framework for ICIs, which includes resilience enhancement strategies hierarchical modelling, decomposition and optimization.

We have constructed a hierarchical model of RES based on a Model Predictive Control-based dynamic modelling framework for ICIs. The factors relevant to RES are decomposed into multiple layers, including strategic goals at different stages of a disruptive event, tactic prin- 
ciples to achieve the goals and practical activities taken to enhance the local performance at subsystem or component-level. The goal of resilience enhancement is decomposed into mitigation and recovery, which are two main system capacities of the protection that refer to two different phases of the accident evolution during disruptive events. Four resilience improvement principles are considered: increasing effectiveness of the system response, reducing the consequence of disruptions, enhancing system recoverability and strengthening organizational preparedness. The practical activities related to these refer to hazard identification, failure detection, vulnerable areas/elements identification, topology modification, allocation of redundancies and storages, staff training, communications within and between CIs, preparedness by users, improving repair efficiency and sequences. The relationships between different factors are identified and analysed.

To quantify the efforts of RESs, the cost functions associated with the activities have been defined. Then, the optimal RESs for ICIs are obtained by solving a MOO problem, which minimizes the cost and maximizes the total resilience $R_{t}$ of ICIs. We have applied the proposed framework to a case study concerning a gas supply system and a power grid. According to the structure and system parameters of the case study, 13 possible RES activities are proposed. A MOO problem is formulated and the optimal configurations of activities, i.e., the optimal RESs, for the ICIs are identified by the NSGA-II algorithm. The results show that the proposed framework provides insights on the RES for ICIs.

For future work, it would be interesting to study more sophisticated cost-parameter relations, i.e., Eqs. (14) (15), as well as the associated uncertainties (i.e., improvements in related system parameters, e.g., link recovery rates, are not necessarily deterministic functions of the invested resources). We believe that statistical learning would be a promising framework to address this problem given that sufficient historical data is available.

\section{References}

[1] Hosseini S, Barker K, Ramirez-Marquez JE. A review of definitions and measures of system resilience. Reliab Eng Syst Saf 2016;145:47-61. 
https://doi.org/10.1016/j.ress.2015.08.006.

[2] Barker K, Lambert JH, Zobel CW, Tapia AH, Ramirez-Marquez JE, Albert L, et al. Defining resilience analytics for interdependent cyber-physical-social networks. Sustain Resilient Infrastruct 2017;2:59-67.

[3] Ramirez-Marquez JE, Rocco CM, Barker K, Moronta J. Quantifying the Resilience of Community Structures in Networks. Reliab Eng Syst Saf 2017. https://doi.org/10.1016/j.ress.2017.09.019.

[4] Fang Y-P, Pedroni N, Zio E. Resilience-Based Component Importance Measures for Critical Infrastructure Network Systems. IEEE Trans Reliab 2016;65:502-12. https://doi.org/10.1109/TR.2016.2521761.

[5] Park J, Seager TP, Rao PSC, Convertino M, Linkov I. Integrating risk and resilience approaches to catastrophe management in engineering systems. Risk Anal 2013;33:35667. https://doi.org/10.1111/j.1539-6924.2012.01885.x.

[6] Henry D, Ramirez-Marquez JE. Generic metrics and quantitative approaches for system resilience as a function of time. Reliab Eng Syst Saf 2012;99:114-22. https://doi.org/10.1016/j.ress.2011.09.002.

[7] Holling CS. Resilience and Stability of Ecological Systems. Annu Rev Ecol Syst 1973;4:1-23. https://doi.org/10.1146/annurev.es.04.110173.000245.

[8] SRA. Society of risk analysis, glossary of the specialty group on foundations of risk analysis 2015:http://www.sra.org/news/sra-develops-glossary-risk.

[9] Francis R, Bekera B. A metric and frameworks for resilience analysis of engineered and infrastructure systems. Reliab Eng Syst Saf 2014;121:90-103. https://doi.org/10.1016/j.ress.2013.07.004.

[10] Ellis J, Fisher D, Longstaff T, Pesante L, Pethia R, Miller TR, et al. Report to the President's Commission on Critical Infrastructure Protection 1997.

[11] Zio E. Reliability Analysis of Systems of Systems. IEEE Reliab Mag Febr 20162016.

[12] Rinaldi SM, Peerenboom JP, Kelly TK. Identifying, understanding, and analyzing critical infrastructure interdependencies. IEEE Control Syst Mag 2001;21:11-25. https://doi.org/10.1109/37.969131.

[13] Buldyrev S V., Parshani R, Paul G, Stanley HE, Havlin S. Catastrophic cascade of failures in interdependent networks. Nature 2010;464:1025-8. https://doi.org/10.1038/nature08932.

[14] Fang Y, Zio E. Game-Theoretic Decision Making for the Resilience of Interdependent Infrastructures Exposed to Disruptions 2019:97-114. https://doi.org/10.1007/978-3030-00024-0_6.

[15] Ouyang M. Review on modeling and simulation of interdependent critical infrastructure systems. Reliab Eng Syst Saf 2014;121:43-60. https://doi.org/10.1016/j.ress.2013.06.040.

[16] Patelli E, Tolo S, George-Williams H, Sadeghi J, Rocchetta R, de Angelis M, et al. OpenCossan 2.0: an efficient computational toolbox for risk, reliability and resilience analysis 2018 . 
[17] Turnquist M, Vugrin E. Design for resilience in infrastructure distribution networks. Environmentalist 2013;33:104-20. https://doi.org/10.1007/s10669-012-9428-z.

[18] Croope S, McNeil S. Improving Resilience of Critical Infrastructure Systems Post Disaster Recovery and Mitigation. Transp Res Rec J Transp Res Board 2011.

[19] Godschalk DR. Urban Hazard Mitigation: Creating Resilient Cities. Nat Hazards Rev 2003;4:136-43. https://doi.org/10.1061/(ASCE)1527-6988(2003)4:3(136).

[20] Adie CE. Holistic disaster recovery: Ideas for building local sustainability after a natural disaster. DIANE Publishing; 2001.

[21] Ouyang M, Dueñas-Osorio L, Min X. A three-stage resilience analysis framework for urban infrastructure systems. Struct Saf 2012;36-37:23-31. https://doi.org/10.1016/j.strusafe.2011.12.004.

[22] Uday P, Marais K. Designing Resilient Systems-of-Systems: A Survey of Metrics, Methods, and Challenges. Syst Eng 2015;18:491-510. https://doi.org/10.1002/sys.21325.

[23] Brown G, Carlyle M, Salmerón J, Wood K. Defending critical infrastructure. Interfaces (Providence) 2006;36:530-44.

[24] Alderson DL, Brown GG, Carlyle WM. Operational models of infrastructure resilience. Risk Anal 2015;35:562-86.

[25] Fang Y, Pedroni N, Zio E. Optimization of Cascade-Resilient Electrical Infrastructures and its Validation by Power Flow Modeling. Risk Anal 2015;35:594-607. https://doi.org/10.1111/risa.12396.

[26] Ouyang M, Fang Y. A Mathematical Framework to Optimize Critical Infrastructure Resilience against Intentional Attacks. Comput Civ Infrastruct Eng 2017;32. https://doi.org/10.1111/mice.12252.

[27] Fang Y, Sansavini G. Optimizing power system investments and resilience against attacks. Reliab Eng Syst Saf 2017;159:161-73.

[28] Lee EE, Mitchell JE, Wallace WA. Restoration of services in interdependent infrastructure systems: A network flows approach. IEEE Trans Syst Man Cybern Part C Appl Rev 2007;37:1303-17. https://doi.org/10.1109/TSMCC.2007.905859.

[29] Fang Y, Sansavini G. Emergence of antifragility by optimum postdisruption restoration planning of infrastructure networks. J Infrastruct Syst 2017;23. https://doi.org/10.1061/(ASCE)IS.1943-555X.0000380.

[30] Fang Y-P, Sansavini G. Optimum post-disruption restoration under uncertainty for enhancing critical infrastructure resilience. Reliab Eng Syst Saf 2019;185:1-11. https://doi.org/10.1016/j.ress.2018.12.002.

[31] Almoghathawi Y, Barker K, Albert LA. Resilience-driven restoration model for interdependent infrastructure networks. Reliab Eng Syst Saf 2019;185:12-23.

[32] Sharma N, Tabandeh A, Gardoni P. Regional resilience analysis: A multiscale approach to optimize the resilience of interdependent infrastructure. Comput Civ Infrastruct Eng 2020;35:1315-30.

[33] Ouyang M, Wang Z. Resilience assessment of interdependent infrastructure systems: 
With a focus on joint restoration modeling and analysis. Reliab Eng Syst Saf 2015;141:74-82.

[34] Liu X, Ferrario E, Zio E. Resilience analysis framework for interconnected critical infrastructures. ASCE-ASME J Risk Uncert Engrg Sys, Part B Mech Engrg 2017;3:110. https://doi.org/10.1115/1.4035728.

[35] Westmark VR. A definition for information system survivability. 37th Annu Hawaii Int Conf Syst Sci 2004 Proc 2004;00:1-10. https://doi.org/10.1109/HICSS.2004.1265710.

[36] Bruneau M, Chang SE, Eguchi RT, Lee GC, O’Rourke TD, Reinhorn AM, et al. A Framework to Quantitatively Assess and Enhance the Seismic Resilience of Communities. Earthq Spectra 2003;19:733-52. https://doi.org/10.1193/1.1623497.

[37] Linkov I, Bridges T, Creutzig F, Decker J, Fox-Lent C, Kröger W, et al. Changing the resilience paradigm. Nat Clim Chang 2014;4:407-9.

[38] Deb K, Pratap A, Agarwal S, Meyarivan TAMT. A fast and elitist multiobjective genetic algorithm: NSGA-II. IEEE Trans Evol Comput 2002:182-97.

[39] Zio E. Critical Infrastructures Vulnerability and Risk Analysis. Eur J Secur Res 2016;1:97-114. https://doi.org/10.1007/s41125-016-0004-2.

[40] Camacho EF, Bordons Alba C. Model Predictive Control. Springer Science \& Business Media; 2013.

[41] Muller G. Fuzzy architecture assessment for critical infrastructure resilience. Procedia Comput Sci 2012;12:367-72. https://doi.org/10.1016/j.procs.2012.09.086.

[42] Nair R, Avetisyan H, Miller-Hooks E. Resilience Framework for Ports and Other Intermodal Components. Transp Res Rec J Transp Res Board 2010;2166:54-65. https://doi.org/10.3141/2166-07.

[43] Cimellaro GP, Reinhorn AM, Bruneau M. Framework for analytical quantification of disaster resilience. Eng Struct 2010;32:3639-49. https://doi.org/10.1016/j.engstruct.2010.08.008.

[44] Sharma N, Tabandeh A, Gardoni P. Resilience analysis: A mathematical formulation to model resilience of engineering systems. Sustain Resilient Infrastruct 2018;3:49-67.

[45] Ayyub BM. Systems resilience for multihazard environments: Definition, metrics, and valuation for decision making. Risk Anal 2014;34:340-55.

https://doi.org/10.1111/risa.12093.

[46] Fiksel J. Designing Resilient, Sustainable Systems. Environ Sci Technol 2003;37:53309. https://doi.org/10.1021/es0344819.

[47] Haimes YY. On the definition of resilience in systems. Risk Anal 2009;29:498-501. https://doi.org/10.1111/j.1539-6924.2009.01216.x.

[48] Jackson S, Ferris TLJ. Resilience principles for engineered systems. Syst Eng 2013;16:152-64. https://doi.org/10.1002/sys.21228.

[49] Maliszewski PJ, Perrings C. Factors in the resilience of electrical power distribution infrastructures. Appl Geogr 2012;32:668-79. https://doi.org/10.1016/j.apgeog.2011.08.001.

[50] Mendonça D, Wallace WA. Factors underlying organizational resilience: The case of 
electric power restoration in New York City after 11 September 2001. Reliab Eng Syst Saf 2015;141:83-91. https://doi.org/10.1016/j.ress.2015.03.017.

[51] Hinkel J. " Indicators of vulnerability and adaptive capacity": Towards a clarification of the science-policy interface. Glob Environ Chang 2011;21:198-208. https://doi.org/10.1016/j.gloenvcha.2010.08.002.

[52] Meliopoulos APS, Cokkinides G, Huang R, Farantatos E, Choi S, Lee Y, et al. Smart Grid Technologies for Autonomous Operation and Control. Smart Grid, IEEE Trans 2011;2:1-10. https://doi.org/10.1109/TSG.2010.2091656.

[53] Deene Y De. Essential characteristics of PGD 2008:1-17.

[54] Dinh LTT, Pasman H, Gao X, Mannan MS. Resilience engineering of industrial processes: Principles and contributing factors. J Loss Prev Process Ind 2012;25:233-41. https://doi.org/10.1016/j.jlp.2011.09.003.

[55] Weichselgartner J. Disaster mitigation: the concept of vulnerability revisited. Disaster Prev Manag 2001;10:85-95. https://doi.org/10.1108/09653560110388609.

[56] Pepyne DL. Topology and cascading line outages in power grids. J Syst Sci Syst Eng 2007;16:202-21. https://doi.org/10.1007/s11518-007-5044-8.

[57] Chang S. Infrastructure Resilience to Disasters. Front Eng Reports Leading-Edge Eng from 2009 Symp 2010;39:176. https://doi.org/10.17226/12821.

[58] Santella N, Steinberg LJ, Parks K. Decision making for extreme events: Modeling critical infrastructure interdependencies to aid mitigation and response planning. Rev Policy Res 2009;26:409-22. https://doi.org/10.1111/j.1541-1338.2009.00392.x.

[59] El-Haram MA, Horner MW. Factors affecting housing maintenance cost. J Qual Maint Eng 2002;8:115-23. https://doi.org/10.1108/13552510210430008.

[60] Farmani R, Walters GA, Savic DA. Trade-off between Total Cost and Reliability for Anytown Water Distribution Network. J Water Resour Plan Manag 2005;131:161-71. https://doi.org/10.1061/(ASCE)0733-9496(2005)131:3(161).

[61] Okasha NM, Frangopol DM. Lifetime-oriented multi-objective optimization of structural maintenance considering system reliability, redundancy and life-cycle cost using GA. Struct Saf 2009;31:460-74. https://doi.org/10.1016/j.strusafe.2009.06.005.

[62] Fu S, Zhang D, Montewka J, Yan X, Zio E. Towards a probabilistic model for predicting ship besetting in ice in Arctic waters. Reliab Eng Syst Saf 2016;155:124-36. https://doi.org/10.1016/j.ress.2016.06.010.

[63] Kuznetsova E, Louhichi R, Zio E, Farel R. Input-output Inoperability Model for the risk analysis of eco-industrial parks. J Clean Prod 2017;164:779-92. https://doi.org/10.1016/j.jclepro.2017.06.250.

[64] Chang S, McDaniels T, Beaubien C. Societal impacts of infrastructure failure interdependencies: building an empirical knowledge based. Proc 2009 Tech Counc Lifeline Earthq Eng Conf Oakland, CA 2009:693-702. https://doi.org/10.1061/41050(357)66.

[65] Rocchetta R, Li YF, Zio E. Risk assessment and risk-cost optimization of distributed power generation systems considering extreme weather conditions. Reliab Eng Syst Saf 
2015;136:47-61. https://doi.org/10.1016/j.ress.2014.11.013.

[66] Kucharavy D, De Guio R. Application of S-shaped curves. Procedia Eng 2011;9:55972 .

[67] Nozick LK, Turnquist MA, Jones DA, Davis RD, Lawton CR. Assessing the performance of interdependent infrastructures and optimising investments. Int J Crit Infrastructures 2005;1:144-54. https://doi.org/10.1504/IJCIS.2005.006116.

[68] Lofberg J. YALMIP : a toolbox for modeling and optimization in MATLAB. 2004 IEEE Int. Conf. Robot. Autom. (IEEE Cat. No.04CH37508), IEEE; 2004, p. 284-9.

[69] Cplex. Cplex Optimization Studio IBM ILOG [Online]. 2015, Available: http://www03.ibm.com/software/products/en/ibmilogcpleoptistud 2015.

[70] Li YF, Sansavini G, Zio E. Non-dominated sorting binary differential evolution for the multi-objective optimization of cascading failures protection in complex networks. Reliab Eng Syst Saf 2013;111:195-205. https://doi.org/10.1016/j.ress.2012.11.002. 